Research Article

Open Access

Special Issue Dedicated to Charles R. Johnson

Carlos M. Saiago*

\title{
Diagonalizable matrices whose graph is a tree: the minimum number of distinct eigenvalues and the feasibility of eigenvalue assignments
}

https://doi.org/10.1515/spma-2019-0025

Received September 19, 2019; accepted December 4, 2019

\begin{abstract}
Considered are combinatorially symmetric matrices, whose graph is a given tree, in view of the fact recent analysis shows that the geometric multiplicity theory for the eigenvalues of such matrices closely parallels that for real symmetric (and complex Hermitian) matrices. In contrast to the real symmetric case, it is shown that (a) the smallest example (13 vertices) of a tree and multiplicity list $(3,3,3,1,1,1,1)$ meeting standard necessary conditions that has no real symmetric realizations does have a diagonalizable realization and for arbitrary prescribed (real and multiple) eigenvalues, and (b) that all trees with diameter $<8$ are geometrically di-minimal (i.e., have diagonalizable realizations with as few of distinct eigenvalues as the diameter). This re-raises natural questions about multiplicity lists that proved subtly false in the real symmetric case. What is their status in the geometric multiplicity list case?
\end{abstract}

Keywords: Assignments; Branch duplication; Combinatorially symmetric; Diagonalizable matrix; Diameter; Eigenvalue; Geometric multiplicity; Graph of a matrix; Tree

MSC: 15A18, 05C05, 05C50

\section{Introduction}

In the last three decades, there has been considerable study of the possible multiplicity lists for the eigenvalues of real symmetric (Hermitian) matrices, whose graph is a given tree (and more general graphs, as well) [1-12, etc.]. The maximum multiplicity is known [1], there is a close lower bound on the minimum number of distinct eigenvalues [2], and methods for getting all multiplicity lists for several classes of trees [3$6,8,9]$. This work, and much more, is included in the new book [13]. In this study, several conjectures naturally arose. They were consistent with known multiplicity lists on smaller numbers of vertices $(<13)$ and with large classes of trees. However, certain of these conjectures have proven false in the general symmetric case (geometric/algebraic multiplicities in real symmetric matrices whose graph is a given tree), though the smallest counterexamples require large numbers of vertices (13 in one case and 16 in another).

Our purpose here is to re-examine these conjectures in a somewhat more general setting in which we consider combinatorially symmetric matrices $A=\left(a_{i j}\right)$, i.e., $a_{i j} \neq 0$ if and only if $a_{j i} \neq 0$. If $A$ is $n$-by- $n$ the graph of $A$ is a (undirected) graph on $n$ vertices $1, \ldots, n$ with an edge $\{i, j\}, i \neq j$, if and only if $a_{i j} \neq 0$. We take the underlying field to be $\mathbb{R}$ or $\mathbb{C}$, which is sufficient for all that we do and all that is of interest to

\footnotetext{
^Corresponding Author: Carlos M. Saiago: Centro de Matemática e Aplicações (CMA/FCT/UNL), and Departamento de Matemática, Faculdade de Ciências e Tecnologia da Universidade NOVA de Lisboa, 2829-516 Quinta da Torre, Portugal, E-mail: cls@fct.unl.pt
} 
us. However, the arguments are typically valid over a "sufficient large" field. $G F_{2}$ is not good enough, as the "branch duplication" we use simply cannot be carried out.

Very recently, it has been shown that much of the multiplicity theory, for real symmetric matrices whose graph is a tree, is also valid for geometric multiplicities in combinatorially symmetric (diagonalizable) matrices whose graph is a given tree [14]. (Not all, however [15].) However, there seems to be just enough flexibility in this more general setting that, at least, the smaller counterexamples mentioned above evaporate. This raises the question of whether some conjectures may actually be correct in the new geometric multiplicity setting (geometric multiplicities in combinatorially symmetric matrices whose graph is a given tree). Here, we examine two of the questions referred to above, re-visit important counterexamples and show explicitly that the trees involved no longer provide counterexamples in the geometric multiplicity setting.

The first of these areas deals with the important technique of "assignments" to construct symmetric matrices, whose graph is a given tree, with feasible multiplicity lists. This method is discussed in a few sources including $[10,16]$. If an assignment (of eigenvalues to sub-trees or submatrices), meeting certain basic requirements, can be made, one would like to know that there is a real symmetric matrix with an associated multiplicity list. Usually there is, and it is known that there is under certain additional hypotheses $[13,16]$. However, there is a counterexample in general, the smallest having 13 vertices $[13,16]$. The only assignment that would achieve the multiplicities 3, 3, 3,1,1,1,1 for that tree is infeasible in the symmetric case for subtle reasons. We explicitly show here that this assignment is geometrically realizable by a diagonalizable combinatorially symmetric matrix.

The second area is the minimum number of distinct eigenvalues for a matrix, whose graph is a given tree $[2,6,10]$. In both, the symmetric case and geometric multiplicity setting (but not the non-diagonalizable case), this number is known to be at least the "diameter" (measured as the number of vertices in a longest induced path) of the tree $[2,14]$. The trees for which this number is attained in the symmetric case are called di-minimal. It is known that all trees of diameter $d<7$ are di-minimal [10, 13]. But, for diameter $d=7$, there are three families [10] of trees that include non-di-minimal trees. (Even for $d=7$, many trees are di-minimal.) Here, we show that for diameter $d=7$, the known counterexamples ("smallest" non-di-minimal trees) are actually geometrically di-minimal (i.e., there are diagonalizable combinatorially symmetric matrices whose graph is such tree, with only the diameter many distinct real eigenvalues). Explicit examples are constructed.

Our constructions exhaust the known particular counterexamples (and more). This raises the question of whether all trees are geometrically di-minimal (we guess not) and whether all assignments to trees are geometrically realizable.

Note that, when we use the modifier "geometrically" we refer to combinatorially symmetric, diagonalizable matrices whose graph is a given tree, rather than real symmetric or (equivalently) Hermitian matrices.

\section{Definitions and Branch Duplication}

Given a graph $G$ on $n$ vertices and a matrix $A$ whose graph is $G$, if $\alpha$ is an index subset of $\{1, \ldots, n\}$ then $A(\alpha)$ (resp. $G(\alpha)$ ) denotes the principal submatrix of $A$ (resp. induced subgraph of $G$ ) resulting from deletion of the rows and columns (resp. vertices) indexed by $\alpha$, and $A[\alpha]$ (resp. $G[\alpha]$ ) denotes the principal submatrix of $A$ (resp. induced subgraph of $G$ ) resulting from keeping only the rows and columns (resp. vertices) indexed by $\alpha$. If $G^{\prime}=G[\alpha]$ we often write $A\left[G^{\prime}\right]$, meaning the principal submatrix $A[\alpha]$. We abbreviate $A(\{i\})($ resp. $G(\{i\}))$ by $A(i)$ (resp. $G(i)$ ). When $G$ is a tree, $A(i)$ is a direct sum, whose summands correspond to components of $G(i)$, which we call branches of $G$ at $v$.

Given an $n$-by- $n$ matrix $A$ we denote by $p_{A}(t)$ the characteristic polynomial of $A$ and we denote by $\operatorname{am}_{A}(\lambda)$ (resp. $\operatorname{gm}_{A}(\lambda)$ ) the algebraic (resp. geometric) multiplicity of $\lambda$ as an eigenvalue of $A$.

We now describe the process of branch duplication for combinatorially symmetric matrices whose graph is a tree and first we give a combinatorial version of it.

Let $T$ be a tree and $\left\{v, u_{1}\right\}$ be an edge of $T$. Let $T_{v}$ (resp. $T_{1}$ ) be the connected component of $T$ resulting from deletion of $u_{1}$ (resp. $v$ ) and containing $v$ (resp. $u_{1}$ ). 
An s-combinatorial branch duplication of $T_{1}$ at $v$ results in a new tree in which $s \geq 1$ copies of $T_{1}$ are appended to $T$ at $v$.

Let $A=\left(a_{i j}\right)$ be a combinatorially symmetric matrix whose graph is $T$. By permutation similarity, $A$ is similar to a matrix

$$
\left[\begin{array}{c|c}
\hline A\left[T_{v}\right] & a_{v u_{1}} \\
\hline a_{u_{1} v} & A\left[T_{1}\right] \\
\hline
\end{array}\right.
$$

in which $a_{v u_{1}}$ and $a_{u_{1} v}$ are the nonzero entries of $A$ corresponding to the edge $\left\{v, u_{1}\right\}$ of $T$. Without loss of generality we assume the above form for matrix $A$.

Let $\breve{T}$ be a tree obtained from $T$ by an $s$-combinatorial branch duplication of $T_{1}$ at $v$. We denote by $u_{2}, \ldots, u_{1+s}$ (resp. $T_{2}, \ldots, T_{1+s}$ ) the new neighbors of $v$ (resp. the new branches at $v$ ) in $\check{T}$. We say that a matrix $\check{A}=\left(\check{a}_{i j}\right)$ is obtained from $A$ by an s-algebraic branch duplication of summand (branch) $A\left[T_{1}\right]$ at $v$ if the graph of $\check{A}$ is $\check{T}$ and $\check{A}$ satisfies the following requirements (i) and (ii):

$$
\begin{gathered}
\text { (i) } \check{A}\left[T_{v}\right]=A\left[T_{v}\right] \text { and } \check{A}\left[T_{1}\right]=\cdots=\check{A}\left[T_{1+s}\right]=A\left[T_{1}\right] \text {; } \\
\text { (ii) } \check{a}_{v u_{i}} \check{a}_{u_{i} v} \neq 0, i=1, \ldots, 1+s \text {, and } \\
\check{a}_{v u_{1}} \check{a}_{u_{1} v}+\cdots+\check{a}_{v u_{1+s}} \check{a}_{u_{1+s} v}=a_{v u_{1}} a_{u_{1} v} .
\end{gathered}
$$

By construction of $\check{A}$ we have

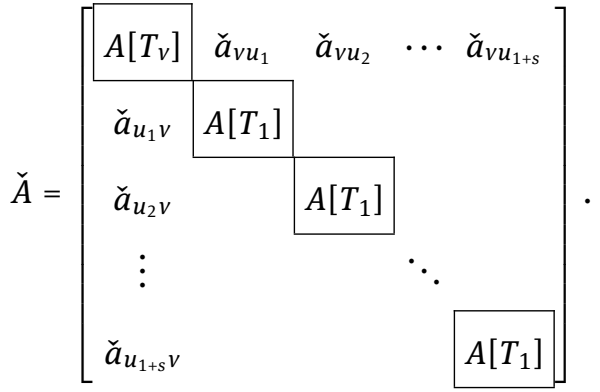

An important property of matrix $\check{A}$ is that the eigenvalues of $\check{A}$ are all those of $A$, together with those corresponding to the duplicated summand (branch) $A\left[T_{1}\right]$, including algebraic and geometric multiplicities, which can be stated as follows.

Theorem 1. Let $T$ be a tree, $v$ a vertex of $T, T_{1}$ a branch of $T$ at $v$ and $A$ be a combinatorially symmetric matrix whose graph is $T$. If $\check{A}$ is obtained from $A$ by an s-algebraic branch duplication of summand $A\left[T_{1}\right]$ at $v$ then $\check{A}$ is similar to the block diagonal matrix $A \oplus_{i=1}^{S} A\left[T_{1}\right]$. Therefore

$$
p_{\check{A}}(t)=p_{A}(t) \cdot\left[p_{A\left[T_{1}\right]}(t)\right]^{s}
$$

and, for each eigenvalue $\lambda$ of $\check{A}$, we have

$$
\operatorname{am}_{\check{A}}(\lambda)=\operatorname{am}_{A}(\lambda)+s \cdot \operatorname{am}_{A\left[T_{1}\right]}(\lambda) \text { and } \operatorname{gm}_{\check{A}}(\lambda)=\operatorname{gm}_{A}(\lambda)+s \cdot \operatorname{gm}_{A\left[T_{1}\right]}(\lambda) .
$$

Proof. Without loss of generality we assume that matrix $A$ has the form (1) and $\check{A}$ has the form (2). Setting $A_{0}=A\left[T_{v}\right], A_{1}=A\left[T_{1}\right], a=a_{v u_{1}}$ and $b=a_{u_{1} v}$, we have

$$
A=\left[\begin{array}{cc}
A_{0} & a \\
b & A_{1}
\end{array}\right] .
$$

Setting $a_{i}=\check{a}_{v u_{i}}$ and $b_{i}=\check{a}_{u_{i} v}, i=1, \ldots, 1+s$, matrix $\check{A}$ is displayed as

$$
\check{A}=\left[\begin{array}{ccccc}
A_{0} & a_{1} & a_{2} & \cdots & a_{1+s} \\
b_{1} & A_{1} & & & \\
b_{2} & & A_{1} & & \\
\vdots & & & \ddots & \\
b_{1+s} & & & & A_{1}
\end{array}\right]
$$


in which, by (ii), we have $a_{1} b_{1}+\cdots+a_{s} b_{s}+a_{1+s} b_{1+s}=a b \neq 0$. By permutation similarity suppose, without loss of generality, that $a_{s} b_{s}+a_{1+s} b_{1+s} \neq 0$.

We prove that $\check{A}$ is similar to the block diagonal matrix $A \oplus_{i=1}^{S} A_{1}$. The remaining conclusions are consequence of the similarity between $\check{A}$ and $A \oplus_{i=1}^{S} A_{1}$, and because $A \oplus_{i=1}^{S} A_{1}$ has that block diagonal structure.

We argue by induction on the number $s \geq 1$ of duplications of summand $A_{1}$.

If $s=1$ then

$$
\check{A}=\left[\begin{array}{ccc}
A_{0} & a_{1} & a_{2} \\
b_{1} & A_{1} & \\
b_{2} & & A_{1}
\end{array}\right] .
$$

Let $I_{i}, i=0,1$, denote the identity matrix of the same size as $A_{i}$. Considering the block matrix

$$
P=\left[\begin{array}{c|c|c}
I_{0} & 0 & 0 \\
\hline 0 & \frac{b_{1}}{b} I_{1} & -\frac{a_{2} b_{2} b_{1}}{a b} I_{1} \\
\hline 0 & \frac{b_{2}}{b} I_{1} & \frac{a_{1} b_{1} b_{2}}{a b} I_{1}
\end{array}\right]
$$

we have

$$
P^{-1}=\left[\begin{array}{c|c|c}
I_{0} & 0 & 0 \\
\hline 0 & \frac{a_{1}}{a} I_{1} & \frac{a_{2}}{a} I_{1} \\
\hline 0 & -\frac{1}{b_{1}} I_{1} & \frac{1}{b_{2}} I_{1}
\end{array}\right]
$$

and

$$
P^{-1} \check{A} P=\left[\begin{array}{ccc}
A_{0} & a & 0 \\
b & A_{1} & \\
\hline 0 & & A_{1}
\end{array}\right] .
$$

Thus the claimed result is valid for $s=1$.

Now let $s \geq 2$ and suppose the claimed result valid for $k=s-1$ duplications of summand $A_{1}$. Choose nonzero scalars $a_{s}^{\prime}$ and $b_{s}^{\prime}$ such that $a_{s}^{\prime} b_{s}^{\prime}=a_{s} b_{s}+a_{1+s} b_{1+s}(\neq 0)$ and consider the matrix

$$
B=\left[\begin{array}{ccccc}
A_{0} & a_{1} & \cdots & a_{s-1} & a_{s}^{\prime} \\
b_{1} & A_{1} & & & \\
\vdots & & \ddots & & \\
b_{s-1} & & & A_{1} & \\
b_{s}^{\prime} & & & & A_{1}
\end{array}\right]
$$

obtained from $A$ by an (s - 1)-algebraic branch duplication of summand $A\left[T_{1}\right]$ at $v$. Since $a_{1} b_{1}+\cdots+a_{s-1} b_{s-1}+$ $a_{s}^{\prime} b_{s}^{\prime}=a b$, by the induction hypothesis matrix $B$ is similar to the block diagonal matrix $A \oplus_{i=1}^{s-1} A_{1}$.

Since $a_{s}^{\prime} b_{s}^{\prime}=a_{s} b_{s}+a_{1+s} b_{1+s}$, by the case $s=1$ we may conclude that $\check{A}$ is similar to the block diagonal matrix $B \oplus A_{1}$. Since $B$ is similar to $A \oplus_{i=1}^{s-1} A_{1}$, the result follows.

Note that Theorem 1 could be stated in a more general form, in which $T_{v}$ and $T_{1}$ are general graphs connected by an edge, the bridge $\left\{v, u_{1}\right\}$. The claimed result and proof would be exactly the same as of Theorem 1 . Also note that Theorem 1 extends [6, Theorem 1].

Remark. It is not essential to how we apply Theorem 1, but there is also a converse; condition (ii) is also necessary, even for a common characteristic polynomial in this context. Assume $\check{A}$ has the graph $\check{T}$, and conditions (i) hold. Then if $\check{A}$ and the block diagonal matrix $A \oplus_{i=1}^{S} A\left[T_{1}\right]$ of Theorem 1 have the same characteristic polynomial, the condition (ii) must hold. (Thus, Theorem 1 could have been an "if and only if" statement.) This follows from expansions of both determinants along the row corresponding to vertex $v$ for a value of the argument of the characteristic polynomials for which none of the relevant principal minors is 0 (and equating). This also holds in the generality of the prior paragraph.

We recall now the notion of "seed" and "family" of trees of a given diameter [10]. The result of a sequence of combinatorial branch duplications, starting with a tree $T$ (at possibly different or new vertices $v$ and duplicating possibly different branches) is called an unfolding of $T$. 
By a seed of diameter $d$, we mean a tree of diameter $d$ that is not an unfolding of any tree of diameter $d$ with fewer vertices. The path on $d$ vertices is always a seed of diameter $d$ and every seed of diameter $d$ has this path as its diameter. There are finitely many seeds of diameter $d$, and any tree of diameter $d$ (that is not a seed) is an unfolding of a unique seed of diameter $d$ [17].

We call all the diameter $d$ unfoldings of a diameter $d$ seed the family of that seed. Since each diameter $d$ tree is an unfolding of only one (unique) seed, the families of the diameter $d$ seeds partition the diameter $d$ trees, but each family is, itself, infinite.

\section{Assignments}

Historically, in the symmetric case, an assignment is an allocation of eigenvalues to simple subtrees of a tree of interest in hopes of achieving a desired multiplicity list. If realizable, the basic Parter-Wiener, etc. theory [18] should verify that the larger tree affords the desired multiplicity list. The method is described, for example, in [16] and [13], along with examples, and was used informally long before by the authors and collaborators. With some obvious constraints about the number of eigenvalues assigned, implicitly or explicitly, to a subtree, typically an assignment is realizable. They are always necessary for a given multiplicity list and are sufficient for trees with fewer than 13 vertices. The first example to the contrary is the 13 vertex tree

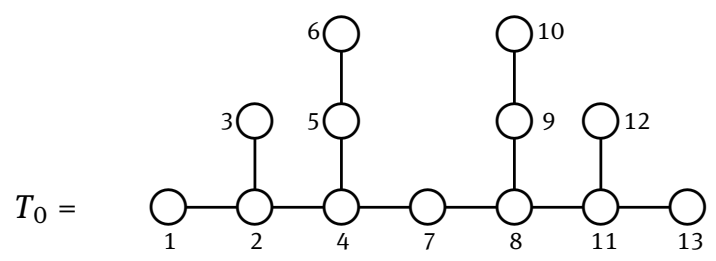

and the desired multiplicity list $(3,3,3,1,1,1,1)$. Despite meeting known conditions, this list is not symmetrically realizable for $T_{0}$ (i.e., there is no real symmetric matrix with graph $T_{0}$ and with such multiplicity list). This was first noticed and analyzed in [16] and is also reported in [13]. The problem is a subtle contradiction to the order of the eigenvalues through interlacing.

However, in the geometric multiplicity setting the displayed assignment for $T_{0}$

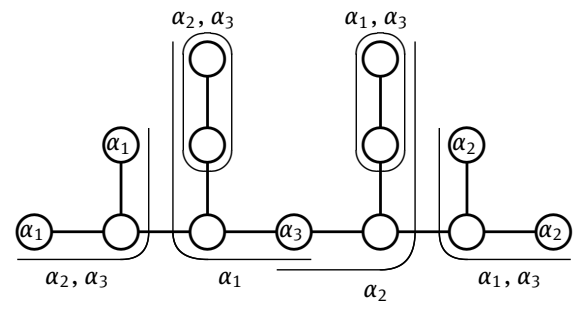


is realizable by the diagonalizable combinatorially symmetric matrix

$$
A=\left[\begin{array}{rrrrrrrrrrrrr}
1 & -1 & 0 & 0 & 0 & 0 & 0 & 0 & 0 & 0 & 0 & 0 & 0 \\
1 & 4 & -1 & 1 & 0 & 0 & 0 & 0 & 0 & 0 & 0 & 0 & 0 \\
0 & 1 & 1 & 0 & 0 & 0 & 0 & 0 & 0 & 0 & 0 & 0 & 0 \\
0 & 1 & 0 & -3 & -2 & 0 & -8 & 0 & 0 & 0 & 0 & 0 & 0 \\
0 & 0 & 0 & 1 & 4 & -2 & 0 & 0 & 0 & 0 & 0 & 0 & 0 \\
0 & 0 & 0 & 0 & 1 & 1 & 0 & 0 & 0 & 0 & 0 & 0 & 0 \\
0 & 0 & 0 & 1 & 0 & 0 & 3 & -3 & 0 & 0 & 0 & 0 & 0 \\
0 & 0 & 0 & 0 & 0 & 0 & 1 & -1 & 2 & 0 & -1 & 0 & 0 \\
0 & 0 & 0 & 0 & 0 & 0 & 0 & 1 & 2 & 1 & 0 & 0 & 0 \\
0 & 0 & 0 & 0 & 0 & 0 & 0 & 0 & 1 & 2 & 0 & 0 & 0 \\
0 & 0 & 0 & 0 & 0 & 0 & 0 & 1 & 0 & 0 & 2 & 2 & -1 \\
0 & 0 & 0 & 0 & 0 & 0 & 0 & 0 & 0 & 0 & 1 & 2 & 0 \\
0 & 0 & 0 & 0 & 0 & 0 & 0 & 0 & 0 & 0 & 1 & 0 & 2
\end{array}\right] .
$$

which has the multiplicity list $(3,3,3,1,1,1,1)$. The prescribed multiplicity 3 eigenvalues are $\alpha_{1}=1, \alpha_{2}=2$ and $\alpha_{3}=3$. (Note that the matrices presented here, and below, begin with an assignment but result in an explicit, checkable example.)

The matrix $A$ realizing the desired assignment of the tree $T_{0}$ was not constructed directly. In fact such a matrix $A$ was obtained, by branch duplication, from a smaller 7-by-7 matrix $A_{0}$ which graph is the path $S_{0}$ on 7 vertices with the displayed assignment

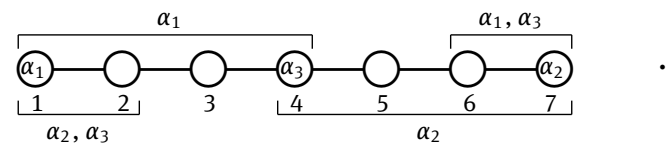

Since $S_{0}$ is a seed of $T_{0}$, if the displayed assignment for $S_{0}$ is realizable in the geometric multiplicity setting, then $A$ (and $T_{0}$ ) will be obtained from $A_{0}$ (and $S_{0}$ ) by branch duplication, in order to have $A$ realizing the above displayed assignment for $T_{0}$. A diagonalizable combinatorially symmetric matrix realizing the displayed assignment for $S_{0}$ is

$$
A_{0}=\left[\begin{array}{rrrrrrr}
1 & -2 & 0 & 0 & 0 & 0 & 0 \\
1 & 4 & -1 & 0 & 0 & 0 & 0 \\
0 & 1 & -3 & -8 & 0 & 0 & 0 \\
0 & 0 & 1 & 3 & -3 & 0 & 0 \\
0 & 0 & 0 & 1 & -1 & 1 & 0 \\
0 & 0 & 0 & 0 & 1 & 2 & 1 \\
0 & 0 & 0 & 0 & 0 & 1 & 2
\end{array}\right]
$$

which has 7 distinct (real) eigenvalues $\alpha_{1}, \ldots, \alpha_{7}$, with $\alpha_{1}=1, \alpha_{2}=2$ and $\alpha_{3}=3$. (Note that here, and below, the vertex numberings are unnecessary for displaying the desired assignment. However, we present matrix $A$ according to the vertex labels shown.)

Of course, the displayed assignment for the path $S_{0}$ is not realizable in the symmetric case. But we also may see that, considering such an assignment in the symmetric case, the highlighted assignment

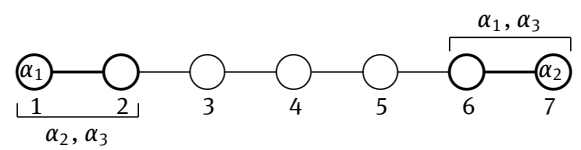

to the subgraph of $S_{0}$ induced by vertices 1 and 2 would imply, by interlacing, that $\alpha_{1}$ is strictly between $\alpha_{2}$ and $\alpha_{3}$. Similarly, the highlighted assignment to the subgraph of $S_{0}$ induced by vertices 6 and 7 would imply, by interlacing, that $\alpha_{2}$ is strictly between $\alpha_{1}$ and $\alpha_{3}$, resulting a contradiction to the order of the assigned eigenvalues $\alpha_{1}, \alpha_{2}$ and $\alpha_{3}$. 


\section{Geometric Di-minimality}

The proof that all trees of a given diameter, or of a large family with fixed diameter, are di-minimal rests on the powerful technique of branch duplication, and a well chosen assignment to a seed tree. This is developed for Hermitian matrices in $[6,10]$ and summarized in [13]. The technique was used to show that all trees with diameter $d<7$ are di-minimal [10] and to identify the only three seeds (of 12) whose families contain non-di-minimal trees. Here, we revisit those three seeds (families).

The smallest example of a non-di-minimal tree has 16 vertices:

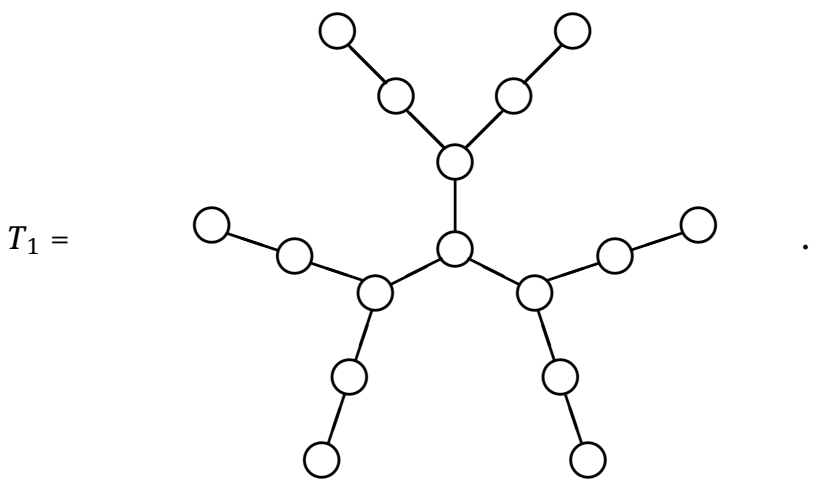

For real symmetric matrices with this graph, there must be at least 8 distinct eigenvalues (and 8 is attainable) $[10,19]$. The tree $T_{1}$ is in the family of the 7 -path seed

$$
S_{1}=
$$

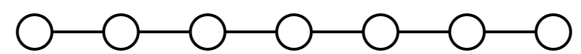

under (combinatorial) branch duplication [10]. The assignment needed to achieve only 7 distinct eigenvalues, in a matrix whose graph is $T_{1}$, is not possible for the path $S_{1}$ in the symmetric case.

The other two trees that are the smallest non-di-minimal ones in their families [10] are

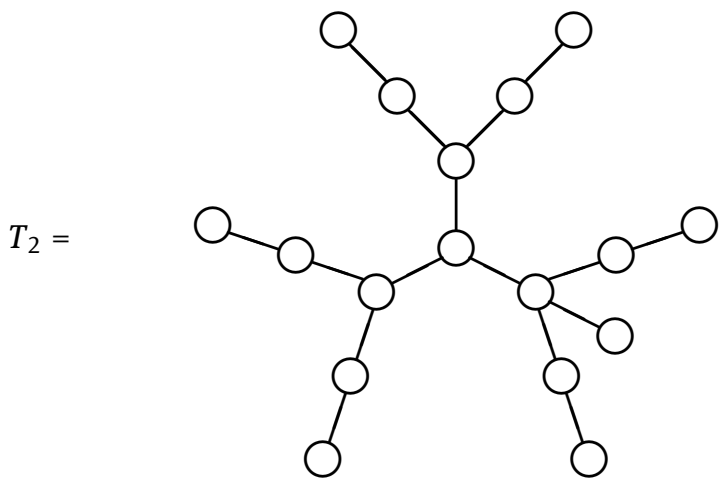

with 17 vertices, from the family of the seed

$$
S_{2}=
$$

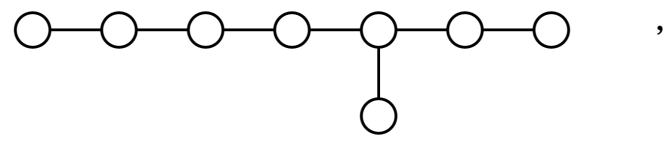

and, with 19 vertices, 


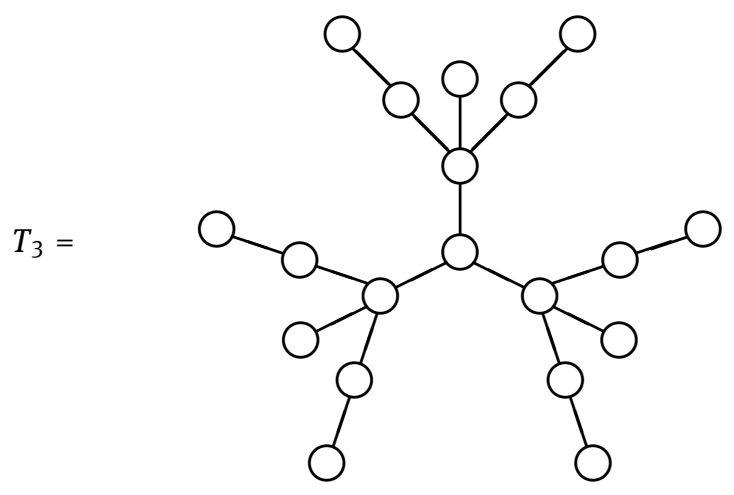

from the family of the seed<smiles>OOOC(O)OOO</smiles>

The example $T_{1}$ was discovered in [19] and explained further in [10]. The minimum number of distinct eigenvalues in real symmetric realizations is 8 . In fact, for trees of diameter 7 , the minimum number of distinct eigenvalues in real symmetric realizations is 7 or 8 [10]. The examples $T_{2}$ and $T_{3}$ were identified in [10], where it was shown that the other nine diameter 7 families are all di-minimal. This fully resolved all cases of diameter $<8$.

First, we show that $T_{1}$ is actually geometrically di-minimal with a simple example.

For the tree $T_{1}$ with the displayed assignment

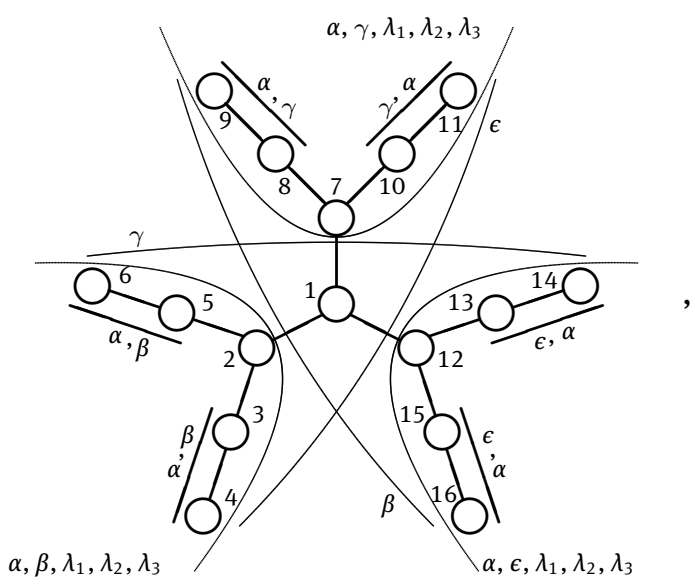


a diagonalizable combinatorially symmetric matrix realizing this assignment is

$$
A=\left[\begin{array}{rrrrrrrrrrrrrrrr}
5 & -25 & 0 & 0 & 0 & 0 & 2 & 0 & 0 & 0 & 0 & 5 & 0 & 0 & 0 & 0 \\
1 & -4 & -4 & 0 & -3 & 0 & 0 & 0 & 0 & 0 & 0 & 0 & 0 & 0 & 0 & 0 \\
0 & 1 & 11 & -54 & 0 & 0 & 0 & 0 & 0 & 0 & 0 & 0 & 0 & 0 & 0 & 0 \\
0 & 0 & 1 & -4 & 0 & 0 & 0 & 0 & 0 & 0 & 0 & 0 & 0 & 0 & 0 & 0 \\
0 & 1 & 0 & 0 & 11 & -54 & 0 & 0 & 0 & 0 & 0 & 0 & 0 & 0 & 0 & 0 \\
0 & 0 & 0 & 0 & 1 & -4 & 0 & 0 & 0 & 0 & 0 & 0 & 0 & 0 & 0 & 0 \\
1 & 0 & 0 & 0 & 0 & 0 & -3 & 1 & 0 & 2 & 0 & 0 & 0 & 0 & 0 & 0 \\
0 & 0 & 0 & 0 & 0 & 0 & 1 & -5 & -60 & 0 & 0 & 0 & 0 & 0 & 0 & 0 \\
0 & 0 & 0 & 0 & 0 & 0 & 0 & 1 & 11 & 0 & 0 & 0 & 0 & 0 & 0 & 0 \\
0 & 0 & 0 & 0 & 0 & 0 & 1 & 0 & 0 & -5 & -60 & 0 & 0 & 0 & 0 & 0 \\
0 & 0 & 0 & 0 & 0 & 0 & 0 & 0 & 0 & 1 & 11 & 0 & 0 & 0 & 0 & 0 \\
1 & 0 & 0 & 0 & 0 & 0 & 0 & 0 & 0 & 0 & 0 & -2 & 1 & 0 & 5 & 0 \\
0 & 0 & 0 & 0 & 0 & 0 & 0 & 0 & 0 & 0 & 0 & 1 & -3 & -24 & 0 & 0 \\
0 & 0 & 0 & 0 & 0 & 0 & 0 & 0 & 0 & 0 & 0 & 0 & 1 & 8 & 0 & 0 \\
0 & 0 & 0 & 0 & 0 & 0 & 0 & 0 & 0 & 0 & 0 & 1 & 0 & 0 & -3 & -24 \\
0 & 0 & 0 & 0 & 0 & 0 & 0 & 0 & 0 & 0 & 0 & 0 & 0 & 0 & 1 & 8
\end{array}\right]
$$

having the eigenvalues $\alpha=5, \beta=2, \gamma=1, \epsilon=0, \lambda_{1}=3, \lambda_{2}=4, \lambda_{3}=-4$ with corresponding multiplicities $4,2,2,2,2,2,2$, respectively.

Now, we may show that each of the families whose seeds are $S_{1}, S_{2}$ and $S_{3}$ is geometrically di-minimal.

For the seed $S_{1}$, the (diagonalizable) assignment

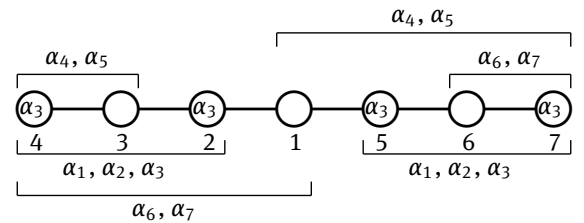

is realizable by the diagonalizable combinatorially symmetric matrix

$$
A_{1}=\left[\begin{array}{rrrrrrr}
0 & 1 & 0 & 0 & 1 & 0 & 0 \\
\frac{180}{7} & 3 & 1 & 0 & 0 & 0 & 0 \\
0 & -6 & 0 & 1 & 0 & 0 & 0 \\
0 & 0 & 4 & 3 & 0 & 0 & 0 \\
-\frac{12}{7} & 0 & 0 & 0 & 3 & 1 & 0 \\
0 & 0 & 0 & 0 & -20 & 0 & 1 \\
0 & 0 & 0 & 0 & 0 & 18 & 3
\end{array}\right] \text {. }
$$

The assigned eigenvalues are $\alpha_{1}=1, \alpha_{2}=2, \alpha_{3}=3, \alpha_{4}=4, \alpha_{5}=-1, \alpha_{6}=6$ and $\alpha_{7}=-3$.

For the seed $S_{2}$, the (diagonalizable) assignment

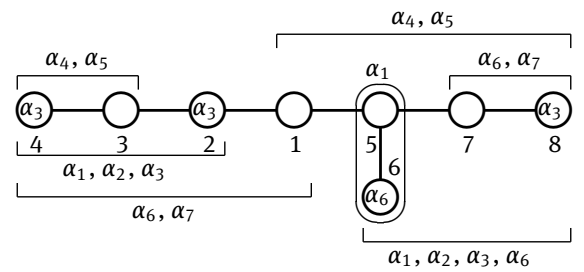


is realizable by the diagonalizable combinatorially symmetric matrix

$$
A_{2}=\left[\begin{array}{rrrrrrrr}
0 & 1 & 0 & 0 & 1 & 0 & 0 & 0 \\
\frac{180}{7} & 3 & 1 & 0 & 0 & 0 & 0 & 0 \\
0 & -6 & 0 & 1 & 0 & 0 & 0 & 0 \\
0 & 0 & 4 & 3 & 0 & 0 & 0 & 0 \\
-\frac{12}{7} & 0 & 0 & 0 & 3 & 1 & 1 & 0 \\
0 & 0 & 0 & 0 & 10 & 6 & 0 & 0 \\
0 & 0 & 0 & 0 & -30 & 0 & 2 & 1 \\
0 & 0 & 0 & 0 & 0 & 0 & 20 & 1
\end{array}\right] .
$$

The assigned eigenvalues are $\alpha_{1}=1, \alpha_{2}=2, \alpha_{3}=3, \alpha_{4}=4, \alpha_{5}=-1, \alpha_{6}=6$ and $\alpha_{7}=-3$, in which $\alpha_{6}$ has multiplicity 2.

Finally, for the seed $S_{3}$, the (diagonalizable) assignment

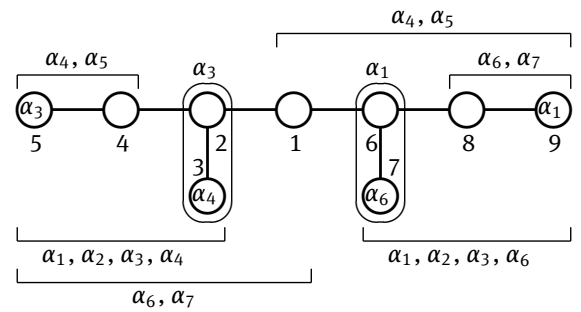

is realizable by the diagonalizable combinatorially symmetric matrix

$$
A_{3}=\left[\begin{array}{rrrrrrrrr}
0 & 1 & 0 & 0 & 0 & 1 & 0 & 0 & 0 \\
\frac{72}{5} & 0 & 1 & 1 & 0 & 0 & 0 & 0 & 0 \\
0 & 3 & 2 & 0 & 0 & 0 & 0 & 0 & 0 \\
0 & -6 & 0 & 1 & 1 & 0 & 0 & 0 & 0 \\
0 & 0 & 0 & 3 & -1 & 0 & 0 & 0 & 0 \\
-\frac{12}{5} & 0 & 0 & 0 & 0 & 0 & 1 & 1 & 0 \\
0 & 0 & 0 & 0 & 0 & 0 & 3 & 0 & 0 \\
0 & 0 & 0 & 0 & 0 & -8 & 0 & 0 & 1 \\
0 & 0 & 0 & 0 & 0 & 0 & 0 & 9 & 0
\end{array}\right] .
$$

The assigned eigenvalues are $\alpha_{1}=0, \alpha_{2}=1, \alpha_{3}=-1, \alpha_{4}=2, \alpha_{5}=-2, \alpha_{6}=3$ and $\alpha_{7}=-3$, in which $\alpha_{4}$ and $\alpha_{6}$ have each multiplicity 2 .

Then, starting from the seed $S_{1}$ (resp. $S_{2}, S_{3}$ ) and matrix $A_{1}$ (resp. $A_{2}, A_{3}$ ) each (combinatorial and algebraic) branch duplication (that does not increase the diameter) has just 7 distinct eigenvalues (the same as those assigned to the seed). In the process of unfolding, by Theorem 1 each matrix obtained from $A_{1}$ (resp. $A_{2}, A_{3}$ ) is diagonalizable. So, the family of the seed $S_{1}$ (resp. $S_{2}, S_{3}$ ) is geometrically di-minimal.

Together with the nine families, for which assignments to seeds were verified in [10], showing (real symmetric) di-minimality, the above examples show that

Theorem 2. All trees of diameter $<8$ are geometrically di-minimal.

We do not know of a tree that is not geometrically di-minimal. Though we would guess that they exist, this raises an obvious question. In any event, there is a significant difference between di-minimality in the geometric multiplicity setting and the symmetric case.

Acknowledgement: This work was carried out within the activities of CMA/FCT/UNL and it was partially supported by the Fundação para a Ciência e a Tecnologia (Portuguese Foundation for Science and Technology) through the project UID/MAT/00297/2019 (Centro de Matemática e Aplicações).

I would like to express my gratitude to Charlie for all these years of friendship and fruitful collaboration. 


\section{References}

[1] C.R. Johnson and A. Leal-Duarte. The maximum multiplicity of an eigenvalue in a matrix whose graph is a tree. Linear and Multilinear Algebra 46:139-144 (1999).

[2] A. Leal-Duarte and C.R. Johnson. On the minimum number of distinct eigenvalues for a symmetric matrix whose graph is a given tree. Mathematical Inequalities and Applications 5(2):175-180 (2002).

[3] C.R. Johnson and A. Leal-Duarte. On the possible multiplicities of the eigenvalues of an Hermitian matrix whose graph is a given tree. Linear Algebra and its Applications 348:7-21 (2002).

[4] C.R. Johnson, A. Leal-Duarte, C.M. Saiago, B.D. Sutton, and A.J. Witt. On the relative position of multiple eigenvalues in the spectrum of an Hermitian matrix with a given graph. Linear Algebra and its Applications 363:147-159 (2003).

[5] C.R. Johnson, A. Leal-Duarte, and C.M. Saiago. Inverse eigenvalue problems and lists of multiplicities of eigenvalues for matrices whose graph is a tree: the case of generalized stars and double generalized stars. Linear Algebra and its Applications 373:311-330 (2003).

[6] C.R. Johnson and C.M. Saiago. Branch duplication for the construction of multiple eigenvalues in an Hermitian matrix whose graph is a tree. Linear and Multilinear Algebra 56(4):357-380 (2008).

[7] C.R. Johnson, B.D. Sutton, and A. Witt. Implicit construction of multiple eigenvalues for trees. Linear and Multilinear Algebra 57(4):409-420 (2009).

[8] C.R. Johnson, J. Nuckols, and C. Spicer. The implicit construction of multiplicity lists for classes of trees and verification of some conjectures. Linear Algebra and its Applications 438(5):1990-2003 (2013).

[9] C.R. Johnson, A.A. Li, and A.J. Walker. Ordered multiplicity lists for eigenvalues of symmetric matrices whose graph is a linear tree. Discrete Mathematics 333:39-55 (2014).

[10] C.R. Johnson and C.M. Saiago. Diameter minimal trees. Linear and Multilinear Algebra 64(3):557-571 (2016).

[11] S.P. Buckley, J.G. Corliss, C.R. Johnson, C.A. Lombardía, and C.M. Saiago. Questions, conjectures, and data about multiplicity lists for trees. Linear Algebra and its Applications, 511:72-109 (2016).

[12] C.R. Johnson and Y. Zhang. Multiplicity lists for symmetric matrices whose graphs have few missing edges. Linear Algebra and its Applications, 540:221-233 (2018).

[13] C.R. Johnson and C.M. Saiago. Eigenvalues, Multiplicities and Graphs. Cambridge Tracts in Mathematics, Cambridge University Press, 2018.

[14] C.R. Johnson and C.M. Saiago. Geometric Parter-Wiener, etc. Theory. Linear Algebra and its Applications, 537:332-347 (2018).

[15] C.R. Johnson, A. Leal-Duarte, and C.M. Saiago. The minimum number of eigenvalues of multiplicity one in a diagonalizable matrix, over a field, whose graph is a tree. Linear Algebra and its Applications 559:1-10 (2018).

[16] C.R. Johnson, C. Jordan-Squire, and D.A. Sher. Eigenvalue assignments and the two largest multiplicities in a Hermitian matrix whose graph is a tree. Discrete Applied Mathematics 158(6):681-691 (2010).

[17] C.R. Johnson, J. Lettie, S. Mack-Crane, and A. Szabelska. Branch duplication in trees: Uniqueness of seed and enumeration of seeds. in manuscript

[18] C.R. Johnson, A. Leal-Duarte, and C.M. Saiago. The Parter-Wiener theorem: refinement and generalization. SIAM Journal on Matrix Analysis and Applications 25(2):352-361 (2003).

[19] F. Barioli and S.M. Fallat. On two conjectures regarding an inverse eigenvalue problem for acyclic symmetric matrices. Electronic Journal of Linear Algebra 11:41-50 (2004).

[20] R. Horn and C.R. Johnson. Matrix Analysis. Cambridge University Press, New York, $2^{\text {nd }}$ Edition, 2013.

[21] S. Parter. On the eigenvalues and eigenvectors of a class of matrices. Journal of the Society for Industrial and Applied Mathematics 8:376-388 (1960).

[22] G. Wiener. Spectral multiplicity and splitting results for a class of qualitative matrices. Linear Algebra and its Applications 61:15-29 (1984). 